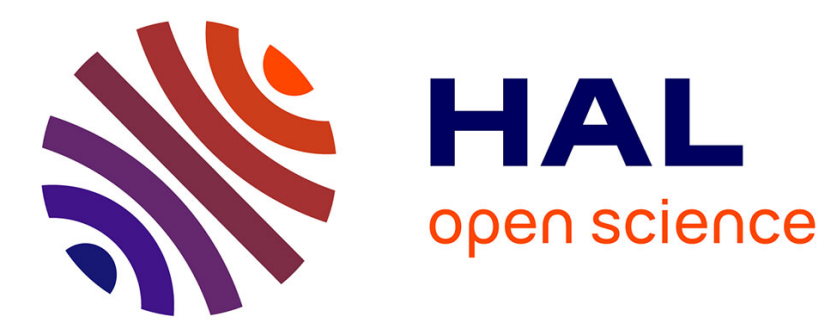

\title{
Learning condyle repositioning during orthognathic surgery with a surgical navigation system
}

\author{
R. Lartizien, I. Zaccaria, C. Savoldelli, L. Noyelles, E. Chamorey, J.L.
}

Cracowski, G. Bettega

\section{To cite this version:}

R. Lartizien, I. Zaccaria, C. Savoldelli, L. Noyelles, E. Chamorey, et al.. Learning condyle repositioning during orthognathic surgery with a surgical navigation system. International Journal of Oral and Maxillofacial Surgery, 2019, 48, pp.952 - 956. 10.1016/j.ijom.2019.01.018 . hal-03486119

\section{HAL Id: hal-03486119 \\ https://hal.science/hal-03486119}

Submitted on 21 Dec 2021

HAL is a multi-disciplinary open access archive for the deposit and dissemination of scientific research documents, whether they are published or not. The documents may come from teaching and research institutions in France or abroad, or from public or private research centers.
L'archive ouverte pluridisciplinaire HAL, est destinée au dépôt et à la diffusion de documents scientifiques de niveau recherche, publiés ou non, émanant des établissements d'enseignement et de recherche français ou étrangers, des laboratoires publics ou privés.

\section{다)(1) $(5$}

Distributed under a Creative Commons Attribution - NonCommerciall 4.0 International 
1 Financial disclosure statement:

2 This research did not receive any specific grant from funding agencies in the public,

3 commercial, or not-for-profit sectors.

4

5 Running heads:

6 Recto: Learning condyle repositioning with a surgical navigation system

7 Verso: Lartizien et al.

8

9 Correspondence:

10 R. Lartizien

11 Maxillofacial and Plastic Surgery Department

12 University Hospital of Grenoble

13 Avenue Maquis-du-Grésivaudan

1438700 La Tronche

15 France

16 E-mail: rodolphe.lartizien@gmail.com

17

18

19

20

21

22

23

24 

navigation system

\section{R. Lartizien ${ }^{1,2,3}{ }^{*}$, I. Zaccaria ${ }^{4}$, C. Savoldelli5, L. Noyelles ${ }^{3}$, E. Chamorey ${ }^{6}$, J.L.}

${ }^{1}$ Maxillofacial and Plastic Surgery Department, University Hospital of Grenoble, Avenue Maquis-du-

Université Grenoble Alpes, Medical Faculty, 23 Avenue Maquis du Grésivaudan, 38700 La Tronche,

34 France

${ }^{3}$ Maxillofacial Surgery Department, Annecy Genevois Hospital, 1 Avenue de l'Hôpital, 74370 Epagny Metz-

Tessy, France

${ }^{6}$ Epidemiology and Biostatistics Unit, Antoine Lacassagne Center, Nice, France

Address:

R. Lartizien

Maxillofacial and Plastic Surgery Department

University Hospital of Grenoble

France 
53 Abstract. Condyle repositioning during bilateral sagittal splint osteotomy (BSSO) is a

54 challenging step for the inexperienced surgeon. We aimed to demonstrate the benefit of

55 navigation for learning the condyle repositioning. We treated 100 patients who

56 underwent a BSSO. A trainee performed the condyle repositioning of one side in two

57 phases. In the first one, the trainee positioned without watching the screen of the

58 Orthopilot Navigation system (ONS). In the second one, the trainee could use the ONS to

59 replace the condyle. Heuristic, anatomical and functional scores of each phase were

60 recorded. Heuristic (17\% vs. $75 \%$; $p<0.0001)$, anatomical (35\% vs. $86 \% ; p<0.0001)$ and

61 functional $(14 \%$ vs. $56 \%$; $p<0.0001)$ scores were significantly greater with the ONS. The

62 ONS is a promising and original intraoperative learning tool for the repositioning of the

63 condyle during BSSO.

64

65 Key words: orthognathic surgery; computer-assisted surgery; condyle repositioning;

66 surgical education; medical education.

67

68

69 
The final result of bilateral sagittal split osteotomies (BSSOs) mainly depends on the correct repositioning of the condyle intraoperatively ${ }^{1}$. For the inexperienced surgeon, condyle repositioning is a challenging step. Many techniques and devices have been described to improve this skill ${ }^{2-7}$ but manual seating remains the method used by the majority of surgeons. The accuracy and reproducibility of this empirical repositioning requires extensive experience and its learning is very complex.

We routinely use in our unit the Orthopilot ${ }^{\mathrm{TM}}$ navigation system (ONS) for orthognathic surgery and particularly for condyle repositioning 2,3 . While this device improved our postoperative occlusal and functional results, its pedagogical interest has not been studied to date.

In this work, our aim was to evaluate the benefit of navigation for learning condyle repositioning during BSSO and to see how resident and inexperienced surgeons acquired this surgical skill.

Materials and methods

This monocentric, observational and retrospective study had included 100 patients needing a BSSO (isolated or associated to a Le Fort I osteotomy), between October 2012 and December 2014. Study ethics approval was obtained on 6 July 2018 (CECIC RhôneAlpes-Auvergne, Clermont-Ferrand, IRB 5891).

\section{The surgical indication was confirmed after clinical examination, sagittal and frontal} cephalometry, and a plaster cast study. The condyle centric relation was recorded preoperatively and documented with a dental splint. The BSSO itself was made by the same experienced surgeon on both sides; on the left side the condyle was repositioned 
and the bicortical screw osteosynthesis was performed by the trainee under supervision of the senior surgeon who positioned and fixed the controlateral condyle. Only the data from the trainee side were considered in this study.

Nineteen trainees were involved in 100 BSSOs. They performed between one and 14 surgical procedures.

\section{Surgical procedure}

The navigation procedure has been described elsewhere ${ }^{2}$. Infrared reflectors (rigid bodies) were anchored via a small vice on each coronoid process. An intermediary fixation between the rigid body and the osseous anchorage allowed the removal and replacement of the rigid body without losing the landmark. Condyle centric relation position was recorded by the system using the specific splint prepared preoperatively and sterilized. BSSOs were performed according to the Obwegeser-Dal Pont technique.

Final occlusion was stabilized by a maxillomandibular fixation. The rigid body was fixed on its vice for condyle navigation. Condyle position was controlled on the computer screen, the surgeon trying to minimize the three translations and the three rotations superimposing the current virtual image of the condyle on the planned one (Fig. 1). The goal was to be as close as possible to zero. The condyle repositioning procedure was conducted in two phases. During the first phase, the trainee manually repositioned the condyle; the screen of the computer was occluded so that he had no access to the navigation data. Once the surgeon believed that the condyle was correctly positioned, the condyle the data were collected by the navigation system and the screen was freed. These data were stocked in a file entitled "without ONS".

During the second phase, the trainee tracked the position on the computer screen and replaced the condyle minimizing the values of rotation and translation. When values of 
120 discrepancies were as closed to 0 as possible, the osteosynthesis was performed with

121 three bicortical screws. This second set of data was recorded in a second file entitled

122 "with ONS".

123

124 Statistical analysis

125 The collected data included: (1) seniority and number of intervention for each surgeons;

126 (2) type of surgical procedure (BSSO isolated or associated to a Le Fort I osteotomy,

127 asymmetry, vertical movement); (3) discrepancies from the reference position of the

128 condyle without and with the ONS (translation top/bottom (Ttb), translation back/front

129 (Tbf), translation left/right (Tlr), frontal rotation (Rf), axial rotation (Ra) and sagittal

130 rotation $(\mathrm{Rs}))$.

131

132 The primary outcome was the heuristic score, calculated as follows:

$$
S H=\sqrt{T t b^{2}+T l r^{2}+T b f^{2}+R s^{2}+R a^{2}+R f^{2}}
$$

133 Heuristic score was considered good when $\leq 5$. The secondary outcomes were the

134 anatomical score and the functional score. Anatomical score was defined by Ttb, Tbf and

135 Rs. Functional score was defined by Ra, Rf and Tlr. Anatomical and functional scores

136 were considered good when each value was $\leq 2$.

137 Scores could be both qualitative (good/not good) and quantitative variables (values).

138 Each score was calculated with and without the ONS (paired series). SA, SH and SF with

139 and without the ONS were analysed by a McNemar test for paired series ${ }^{8}$, in order to

140 know whether the condyle repositioning was significantly better with ONS.

142 Results 
143 Fifty-seven patients were operated by isolated BSSO and 43 also had a Le Fort I

144 osteotomy. Seventy-four patients had an asymmetry. A maxillary impaction was carried 145 out on 31 patients.

146 The number of interventions performed by each surgeon is listed in Table 1.

147 The heuristic score was $\leq 5$ and considered as good in $17 \%$ of the procedures without 148 the ONS and in $75 \%$ with the ONS. The anatomical score was $\leq 2$ in $35 \%$ of the 149 procedures without the ONS and in $86 \%$ with the ONS. The functional score was $\leq 2$ in $15014 \%$ of the procedures without the ONS and in 56\% with the ONS. The use of the ONS 151 significantly improved the three scores of condyle repositioning $(p<0.0001)$. Table 2 152 (upper) shows the heuristic scores with and without the ONS: 38 patients had a bad 153 score without the ONS and remained bad with the ONS, 55 patients were bad without 154 the ONS and achieved a good score with the ONS, one patient had a good score without 155 the ONS and became bad with the ONS, and six patients had a good score without the 156 ONS and remained good with the navigation system. The remainder of Table 2 shows 157 the same type of information for anatomical and functional scores.

158 The distribution of the discrepancy of each variable (Ra, Rf, Rs, Tbf, Trl and Ttb) with 159 and without the ONS is listed in Fig. 2. Graphically, mean and the dispersion of the values 160 were greater without the ONS, especially for the Ra and the Rs.

161 Fig. 3 shows the linear relation between the heuristic score with and without the ONS 162 and the number of interventions performed by the surgeon. The ONS seemed to allow a 163 better repositioning, but with increasing numbers of interventions (and experience) the 164 regression lines tended to join. The intersection was around 15 interventions.

Discussion 
167 The surgical education environment has changed in the past few decades with the

168 tightening of the medical legislation (leading to a decreased autonomy of the practice of

169 trainees), demands for patient safety, the development of new technologies and the

170 limitation of working hours ${ }^{9,10}$. This change imposed the development of new surgical

171 educational tools: cadaveric dissection, simulations ${ }^{10}$, videos ${ }^{11}$ and now virtual reality

17212.

173 Our study demonstrated the educational value of the ONS for condyle repositioning

174 during BSSO. The trainees obtained heuristic (17\% vs. 75\%; $p<0.0001)$, anatomical

175 (35\% vs. 86\%; $p<0.0001)$ and functional ( $14 \%$ vs. $56 \% ; p<0.0001)$ scores significantly

176 greater with the ONS. The ONS gave a virtual image of the condyle and of the target (the

177 final position), so for the trainee the good position is better understood, easier to reach

178 and more accurate than with the empirical (blind) positioning.

179 The heuristic score was chosen to evaluate the improvement of the condyle

180 repositioning because it took into account all of the parameters and considered the

181 control of the three dimensions. The anatomical score represented the condyle position

182 in the sagittal plane. We indicated in a previous study that it was the principal factor in

183 the quality of postoperative occlusion and skeletal stability². The functional score

184 reflected the axial and frontal control of the condyle position and was correlated in the

185 same study with better functional results, especially considering postoperative

186 mandibular motion.

187 In the present study, the functional score was the less improved by the ONS. It proved

188 that perfect repositioning even with tool control is not always possible. Sometimes

189 compromises are needed. The discrepancies are generally due to anatomical conflicts

190 between the proximal and the distal bone segment ${ }^{13}$, especially in asymmetric cases.

191 Rigid osteosynthesis may increase this problem. The improvement of the functional 
score required a remodelling of the osseous valves ${ }^{14}$, but this not always possible without damaging the infra-alveolar nerve.

The analysis of the distribution of the values of each direction with and without the ONS showed that the dispersion of the variables was smaller with the ONS with fewer outliers. The use of the ONS could avoid large discrepancies in the repositioning of the condyle. Berger et al. reached the same conclusion on this point ${ }^{4}$. Without the ONS the dispersions were greater, especially for Rs and Ra. These two dimensions were the most difficult to control without the ONS. This could be explained by the lack of landmarks in the exiguous exposition of the bone intra-orally.

The benefit of a condyle positional tool is the subject of a controversial and long-lasting debate among experts. It is not the subject of this work. We only wanted to emphasize the relevance of navigation in learning this difficult task.

Surgical practice is an essential step for the learning of trainees ${ }^{15,16}$ and the use of the ONS can be beneficial for their educational in orthognathic surgery. Indeed, it allows an inexperienced surgeon to perform a critical step of the surgical procedure without compromising patient safety and postoperative results, his actions being watched continuously. Thus, the ONS allowed to inexperienced surgeons to directly acquire and improve skills to replace the condyle to its reference position (whatever the position chosen preoperatively). This point was demonstrated by the representation of the linear relation between the heuristic score and the number of interventions with and without the ONS: with an increasing number of procedures, the trainee improved his heuristic score without the use of the ONS. The lines seemed to cross at around 15 procedures. In other words, a trainee would be able to place the condyle in the right position without using the ONS after 15 procedures with the ONS. Of course, learning can not be 
summarized by a linear relation but it would be interesting to compare this number

217 with those obtained from other learning tools.

218 Training is more efficient if it is multimodal ${ }^{17}$. The ONS is a new way to teach condylar 219 repositioning and is complementary to other existing tools. To our knowledge, it is the 220 only tool that can be used in the operating room.

221 Furthermore, evaluating technical skills and competence of a trainee in the operating 222 room is difficult ${ }^{18}$, especially because the methods are subjective and unreliable. The 223 ONS could be a good way of objectively following the heuristic scores of trainees and 224 thus their progression.

225 Our study presented several limitations. It was a monocentric study and the number of 226 procedures performed by each trainee was variable. Our results were mainly dependent 227 on the scores obtained by the trainee who performed 14 procedures. A multicentric 228 study with a mixed model (taking into account the correlation of the data) is necessary 229 to confirm our results. The side performed by the trainee was not randomized. This 230 would have introduced a bias. The side should be randomized in future explorations.

231 Another bias was the failure to take into account the trainees' dominant hand. Indeed, a 232 right-handed surgeon would find it more difficult to operate on the left side, for 233 example. This bias had little consequence because the trainee only performed the 234 condyle repositioning and the osteosynthesis (and not the entire intervention) so he 235 could use his dominant hand whatever the operated side. Consequently, a learning 236 effect was observed regardless of the operated side or the dominant hand. However, we 237 should take this point into account in the future.

239 In conclusion. the ONS is a promising and original intraoperative learning tool for the 240 repositioning of the condyle during BSSO. Its use allowed trainees to improve their 
241 accuracy and their surgical skills during this challenging step. However, further

242 explorations are needed to confirm these preliminary results.

243

244 Funding

245 None.

246 Competing interests

247 None.

248 Ethical approval

249 Study ethics approval was obtained on 06 July 2018 (CECIC Rhône-Alpes-Auvergne,

250 Clermont-Ferrand, IRB 5891).

251 Patient consent

252 Not required.

253 


\section{REFERENCES}

255 1. Landes CA, Sterz M. Proximal segment positioning in bilateral sagittal split osteotomy: intraoperative controlled positioning by a positioning splint. J Oral Maxillofac Surg 2003;61:1423-1431.

2. Bettega G, Cinquin P, Lebeau J, Raphael B. Computer-assisted orthognathic surgery: clinical evaluation of a mandibular condyle repositioning system. J Oral Maxillofac Surg 2002;60:27-34.

3. Bettega G, Leitner F. [Computer assisted orthognathic surgery: Condyle repositioning.]. Rev Stomatol Chir Maxillofac Chir Orale 2013. Jul 17. doi: 10.1016/j.revsto.2013.06.001.

4. Berger M, Nova I, Kallus S, Ristow O, Eisenmann U, Dickhaus H, Engel M, Freudlsperger C, Hoffmann J, Seeberger R. Electromagnetic navigated condylar positioning after high oblique sagittal split osteotomy of the mandible: a guided

269 5. Luhr HG. The significance of condylar position using rigid fixation in orthognathic surgery. Clin Plast Surg 1989;16:147-156.

6. Beziat JL, Babic B, Ferreira S, Gleizal A. [Justification for the mandibular-maxillary order in bimaxillary osteotomy]. Rev Stomatol Chir Maxillofac. 2009;110:323-326.

7. Veyssiere A, Leprovost N, Ambroise B, Prevost R, Chatellier A, Benateau H. Study of the mechanical reliability of an S-shaped adjustable osteosynthesis plate for bilateral sagittal split osteotomies. Study on 15 consecutive cases. J Stomatol Oral

8. Mc NQ. Note on the sampling error of the difference between correlated 
9. Haluck RS, Krummel TM. Computers and virtual reality for surgical education in the 21st century. Arch Surg. 2000;135:786-792.

10. Reznick RK, MacRae H. Teaching surgical skills—changes in the wind. N EnglJ Med. 2006;355:2664-2669.

11. Mota $\mathrm{P}$, Carvalho N, Carvalho-Dias E, Joao Costa M, Correia-Pinto J, Lima E. Videobased surgical learning: improving trainee education and preparation for surgery. J Surg Educ 2018;75:828-835.

12. Pulijala $Y$, Ma M, Pears $M$, Peebles D, Ayoub A. An innovative virtual reality training tool for orthognathic surgery. Int J Oral Maxillofac Surg 2018;47:11991205.

13. Suojanen J, Leikola J, Stoor P. The use of patient-specific implants in orthognathic surgery: A series of 30 mandible sagittal split osteotomy patients. J Craniomaxillofac Surg 2017;45:990-994.

14. Ellis E, 3rd. A method to passively align the sagittal ramus osteotomy segments. $J$ Oral Maxillofac Surg 2007;65:2125-2130.

15. Kotsis SV, Chung KC. Application of the "see one, do one, teach one" concept in surgical training. Plast Reconstr Surg 2013;131:1194-1201.

16. Sutkin G, Littleton EB, Kanter SL. Intelligent cooperation: A framework of pedagogic practice in the operating room. Am J Surg 2018;215:535-541.

17. Kim S, Dunkin BJ, Paige JT, Eggerstedt JM, Nicholas C, Vassilliou MC, Spight DH, Pliego JF, Rush RM, Lau JN, Carpenter RO, Scott DJ. What is the future of training in surgery? Needs assessment of national stakeholders. Surgery 2014;156:707717.

18. Winckel CP, Reznick RK, Cohen R, Taylor B. Reliability and construct validity of a structured technical skills assessment form. Am J Surg 1994;167:423-427. 
Table 1. Number of interventions by surgeon

\begin{tabular}{|c|c|c|c|c|}
\hline $\begin{array}{c}\text { Number of } \\
\text { interventions }\end{array}$ & $\begin{array}{r}\text { Number of } \\
\text { surgeons }\end{array}$ & Percentage & $\begin{array}{c}\text { Cumulated } \\
\text { number of } \\
\text { surgeons }\end{array}$ & $\begin{array}{l}\text { Cumulated } \\
\text { percentage }\end{array}$ \\
\hline 1 & 4 & 21.05 & 4 & 21.05 \\
\hline 2 & 3 & 15.79 & 7 & 36.84 \\
\hline 3 & 1 & 5.26 & 8 & 42.11 \\
\hline 4 & 2 & 10.53 & 10 & 52.63 \\
\hline 5 & 2 & 10.53 & 12 & 63.16 \\
\hline 6 & 1 & 5.26 & 13 & 68.42 \\
\hline 8 & 2 & 10.53 & 15 & 78.95 \\
\hline 9 & 1 & 5.26 & 16 & 84.21 \\
\hline 11 & 1 & 5.26 & 17 & 89.47 \\
\hline 13 & 1 & 5.26 & 18 & 94.74 \\
\hline 14 & 1 & 5.26 & 19 & 100.00 \\
\hline
\end{tabular}

305

306 
Table 2. Agreement of each score with and without Orthopilot Navigation system (ONS)

308

\begin{tabular}{|l|l|l|c|}
\hline \multicolumn{3}{|l|}{ Heuristic score (HS) } \\
\hline HS without ONS & \multicolumn{3}{|c|}{ HS with ONS } \\
\hline$\%$ & Bad & Good & Total \\
\hline Bad & 38 & 55 & 93 \\
\hline Good & 1 & 6 & 7 \\
\hline Total & 39 & 61 & 100 \\
\hline Anatomit & & & \\
\hline
\end{tabular}

Anatomical score (AS)

\begin{tabular}{|l|l|l|l|}
\hline AS without ONS & \multicolumn{3}{|c|}{ AS with ONS } \\
\hline$\%$ & Bad & Good & Total \\
\hline Bad & 12 & 53 & 65 \\
\hline Good & 2 & 33 & 35 \\
\hline Total & 14 & 86 & 100 \\
\hline Functional score & & & \\
\hline
\end{tabular}

Functional score (FS)

\begin{tabular}{|l|l|l|c|}
\hline FS without ONS & \multicolumn{3}{|c|}{ FS with ONS } \\
\hline$\%$ & Bad & Good & Total \\
\hline Bad & 41 & 44 & 85 \\
\hline Good & 4 & 11 & 15 \\
\hline Total & 45 & 55 & 100 \\
\hline
\end{tabular}




\section{CAPTIONS TO ILLUSTRATIONS}

311 Fig. 1. Navigation system software interface.

312 Fig. 2. Distribution of the discrepancies of each dimension with and without Orthopilot

313 Navigation system (ONS).

314 Fig. 3. Linear representation between the heuristic score and the number of

315 interventions with and without Orthopilot Navigation system (ONS).

316 

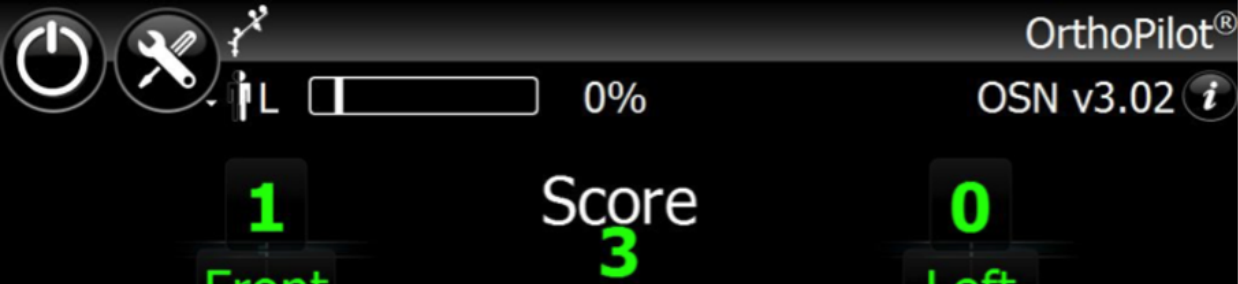

\section{Front}

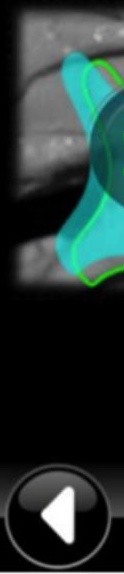

$1^{\circ}$ Anterior 1 Top

$$
\text { (0) } 2^{\circ} \text { Left }
$$

Navigation : Left Condyle

\section{0 Left} OSN v3.02 $\boldsymbol{i}$
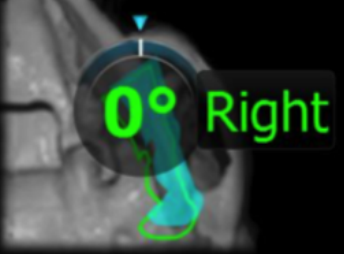
Box-Plot : Discrepancies with/without OSN

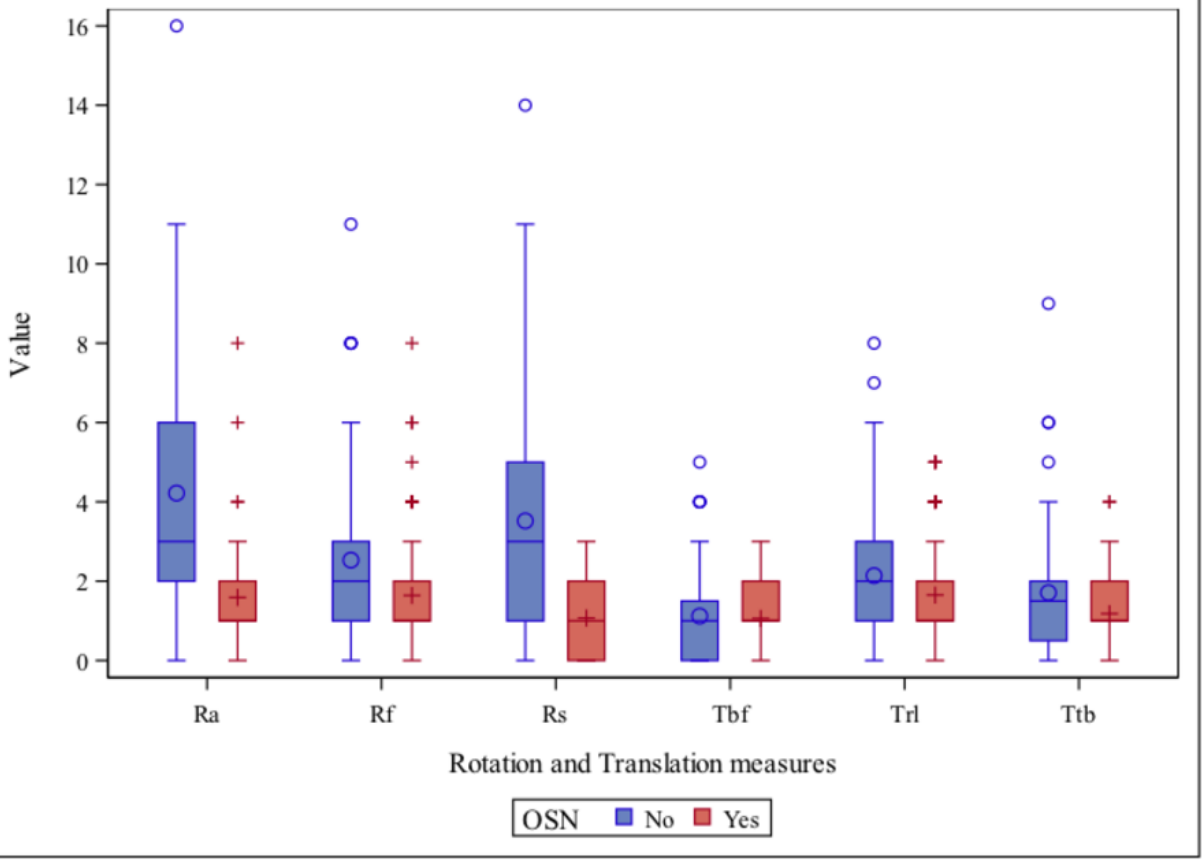




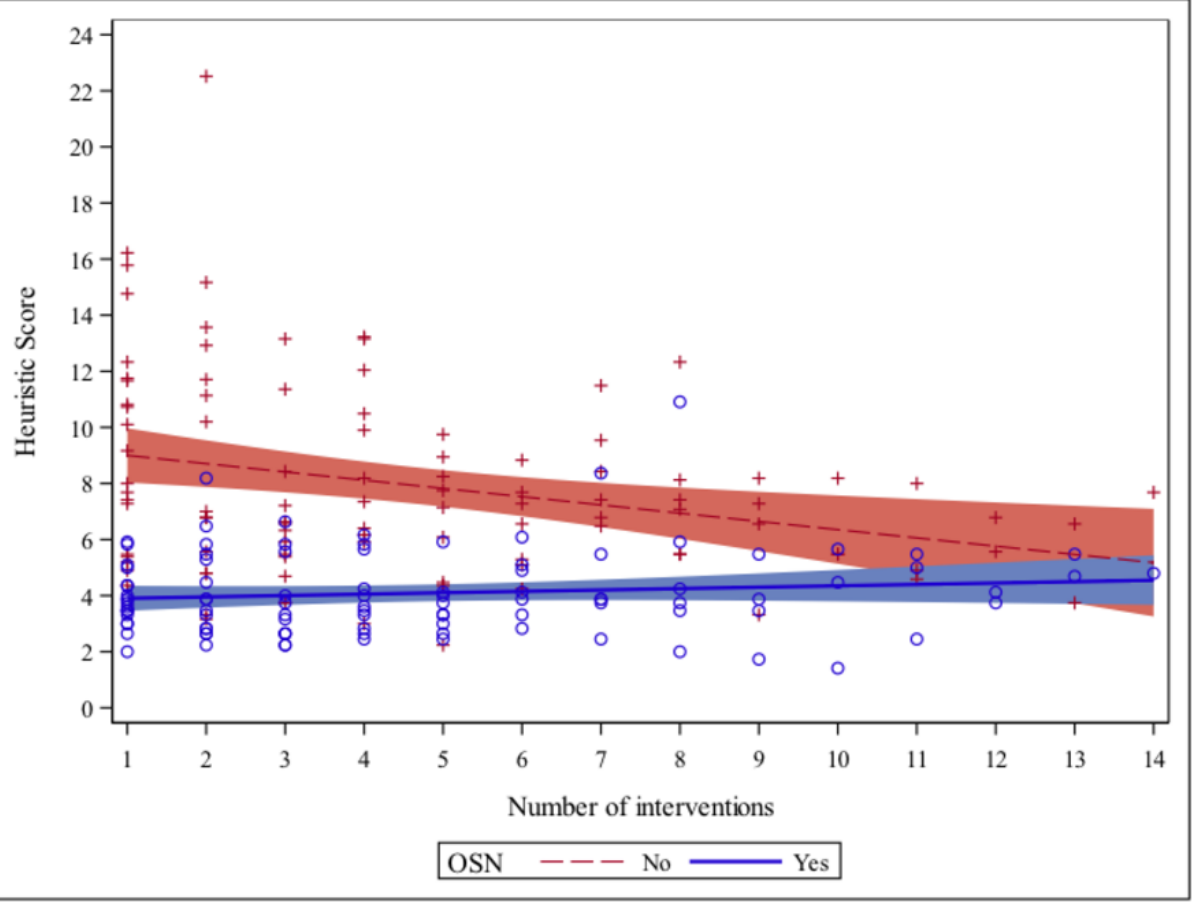

